\title{
¿ES LA LÓGICA UN BUEN INSTRUMENTO CIENTÍFICO PARA HACER FILOSOFÍA?
}

\section{IS LOGIC A GOOD SCIENTIFIC INSTRUMENT FOR DOING PHILOSOPHY?}

\author{
David Suárez-Rivero \\ Profesor visitante. \\ Universidad Nacional, Costa Rica. \\ darisua@gmail.com/dsuarezr@uvaq.edu.mx
}

Recibido: 29 de setiembre / Aprobado: 4 de octubre / Publicado: 28 de octubre de 2021

Resumen: En el presente artículo, ofrezco razones para mostrar que el objeto de estudio de la filosofía son los argumentos, sean epistemológicos, políticos, éticos, estéticos, ontológicos, religiosos, bioéticos, metafísicos, entre otros. Sostengo que su objetivo es formularlos, reconstruirlos, proponerlos y evaluarlos usando la lógica. Presento algunos contraejemplos, brindando una respuesta para mostrar que no debilitan mi propuesta.

Palabras clave: Lógica. Filosofía. Argumento. Evaluación.

\begin{abstract}
In this paper, I offer reasons to show that the goal of Philosophy are arguments, whatever these can be, such as epistemological, political, ethical, aesthetic, ontological, religious, bioethical, metaphysical, among others. I defend that its main objective is to pose, rebuilt, propose, and evaluate arguments using the modern logic. I also present some counter examples, showing that these do not weak my proposal.
\end{abstract}

Keywords: Logic. Philosophy. Argument. Assessment.

\section{Introducción}

Si nos preguntan cuál es el objeto de estudio y qué instrumentos usan científicas, científicos o profesionales, podemos proporcionar fácilmente algunos de ellos, siendo algo inexpertas o inexpertos. Por ejemplo, podemos decir que algunas economistas estudian el mercado regional y mundial, 
usando la probabilidad para predecir su alza o baja; que algunas psicólogas están interesadas en el comportamiento humano, tratando de modificarlo a partir de modelos mentales, utilizando cierta clase de experimentos; o, también, que las químicas investigan elementos físicos para manipularlos y crear nuevos materiales, empleando el método experimental.

¿Qué sucede si nos preguntan, sin embargo, cuál es el objeto de estudio de filósofas y filósofos, y qué instrumentos usan? Quizá, una persona inexperta se quedaría sin respuesta. Entre la comunidad filosófica, si la respuesta a ambas preguntas es polémica, la segunda lo es mucho más. Así, independientemente de cuál sea su objeto de estudio, ¿dicha comunidad usa instrumentos? Alguien podría decir que su herramienta es la reflexión y su objeto de estudio es el pensamiento. Entonces, una respuesta preliminar es que filósofas y filósofos reflexionan sobre el pensar. Mas, ¿qué quiere decir esto?

Planteemos lo anterior de otra manera. Sabemos que las economistas pueden recibir fondos monetarios para predecir en qué situaciones una nación podría estar en quiebra; que cierta clase de psicólogas puede ser incorporada al sistema de salud para ofrecer un servicio a la población, remuneradamente; o que a las químicas se les otorga presupuesto para mutar genes. Si filósofas y filósofos reciben alguna clase de estipendio, ¿lo justifican diciendo que es para reflexionar sobre el pensar? Sería poco útil aseverar esto. Han de poder justificar cuál es su objeto de estudio y qué instrumento usan, así como a las demás disciplinas les es posible hacerlo.

En el presente artículo, en las primeras cinco secciones, ofrezco razones para mostrar que el objeto de estudio de la comunidad filosófica son los argumentos, cualquier clase de ellos, sean epistémicos, éticos, estéticos, ontológicos, metafísicos, religiosos, políticos, sociales, ambientales, económicos o de género. Asimismo, proporciono razones, algunas de ellas formales, en los dos últimos apartados, para mostrar que la lógica —en principio, la monotónica - es un buen instrumento para evaluarlos, haciendo de ellos buenos argumentos. A lo largo del texto, considero también ciertas objeciones, mostrando que no socavan mis razones. 


\section{¿Qué hace la filosofía?}

Pensemos en Platón, en sus Diálogos ${ }^{1}$. La estructura que tienen es la siguiente. Normalmente, Sócrates dialoga con diversos interlocutores sobre diferentes tópicos. Con su docta ignorancia, él formula preguntas y ellos responden. Casi siempre, sin embargo, va encontrando deficiencias en las explicaciones. Por esto, Sócrates les muestra lo erróneo de lo que dicen o la falta de fundamento que hay en su discurso. Como consecuencia, los interlocutores reformulan su discurso u otro interlocutor propone uno nuevo, el cual Sócrates derrumba.

Si alguien nos pregunta ¿qué hace la filosofía?, con los Diálogos de Platón podríamos dar una respuesta. Si pensamos en su estructura, podemos apreciar que Sócrates y sus interlocutores dialogan sistemáticamente. Sócrates formula una pregunta, los otros responden. Sócrates objeta sus respuestas, los interlocutores o reformulan o proponen una nueva. Estas respuestas y objeciones son argumentos, los cuales pueden extraerse para ser estudiados y evaluados por cualquiera. Por ejemplo, Platón proporciona en el Teetetes $(201 \mathrm{~cd})$ el siguiente argumento clásico sobre el conocimiento:

$S$ sabe que $p$ si y solo si:

\section{1. $S$ cree que $\mathrm{p}$,}

2. "p" es verdadera,

3. $S$ tiene razones suficientes para creer que $p$.

Este argumento es conocido como el esquema tripartito del conocimiento, el cual es aceptado y polemizado en nuestros días por toda aquella y aquel que haga epistemología ${ }^{2}$.

¿Qué hace, entonces, la filosofía? La respuesta es sencilla, a saber: propone y evalúa argumentos sistemáticamente. Como a Sócrates, a la comunidad filosófica le interesa conocer qué argumentos hay para, por ejemplo, responder y evaluar lo siguiente: ¿Cuándo podemos decir que la acción de una persona es solidaria y no interesada? ¿Qué criterios hay para discernir entre una acción violenta y otra que no lo es? ¿Cuándo podemos atribuirle a una persona conocimiento y no sabiduría? ¿Qué es lo que hace que ciertas

1 Cooper, John M. (ed.). Plato: Complete Works. Indianapolis: Hackett. 1997.

2 Una versión del esquema puede encontrarse en: Villoro, Luis. Creer, saber y conocer. México: Siglo XXI. 1982. 
oraciones declarativas sean verdaderas y otras falsas? ¿Cuáles son los fundamentos de la necesidad lógica o matemática? ¿A partir de qué momento se puede determinar que un ser vivo es una persona y cuándo deja de serlo? Etc.

Los argumentos, en suma, son el objeto de estudio de la filosofía. Una vez identificados, a filósofas y filósofos les interesa evaluarlos, creando un diálogo sistematizado.

\section{¿La filosofía trabaja solamente con argumentos?}

Alguien podría objetar que no toda filosofía se dedica a proponer y evaluar argumentos. Friedrich Nietzsche, por mencionar a alguno, es un filósofo que podría no ser incluido en el diálogo platónico sistematizado. De hecho, él propone una nueva forma de hacer filosofía, a través del aforismo, evitando reconstruir o evaluar argumentos.

Un ejemplo se puede encontrar en Asi habló Zaratustra, en el apartado "De las tres transformaciones": "Tres transformaciones del espíritu os menciono: cómo el espíritu se convierte en camello, y el camello en león, y el león, por fin, en niño"’.

El aforismo es un método con el que, a través de ciertas imágenes o alegorías, Nietzsche va mostrando sus pensamientos breves respecto al superhombre, a la muerte de dios, la desvalorización de los valores, la ciencia como una nueva forma de religión ${ }^{4}$, etc. La idea de Nietzsche es múltiple y rica, a saber: proponer un método diferente de trasmitir pensamientos, el cual no los manipule, sino que los potencie, para que otros puedan aprehenderlos mucho más genuinamente.

Por lo tanto, la filosofía no solamente propone y evalúa argumentos, sino que también, como Nietzsche, propone imágenes breves que muestran ideas para tener nuevas concepciones sobre el mundo, la cultura, la sociedad, el conocimiento, la religión, la ciencia, etc.

\section{¿Puede traducirse a argumentos otras propuestas metodológicas?}

Sin duda alguna, en la historia de la filosofía, ha habido metodologías diferentes. No solamente Nietzsche propuso una, igual lo han hecho otros.

3 Nietzsche, Friedrich. Asi habló Zaratustra, Madrid: Alianza. 1972, p. 53.

4 En las siguientes obras, pueden encontrarse estos temas: Nietzsche, Friedrich. The Antichrist. New York: Viking. 1954 (1895); Thus Spoke Zarathustra. New York: Viking. 1954 (1883-85); The Gay Science. New York: Vintage. 1974 (1882). 
Edmund Husserl, por ejemplo, propuso la fenomenología ${ }^{5}$; George W. F. Hegel, la dialéctica ${ }^{6}$; George Gadamer, la hermenéutica ${ }^{7}$, o, en Latinoamérica, Mauricio Beuchot, la hermenéutica analógica ${ }^{8}$ ¿Esto significa que el objeto de estudio de filósofas y filósofos no son argumentos?

En absoluto. Todas ellas y todos ellos, con métodos diversos, proponen y evalúan argumentos. Un ejemplo lo podemos encontrar en el libro de Michael Bruce y Steven Barbone ${ }^{9}$, el cual muestra cien argumentos diferentes de la historia de la filosofía occidental, comenzando por la filosofía de la religión, pasando por la metafísica, la epistemología y la ética, y concluyendo con la filosofía de la mente, la filosofía de la ciencia y la filosofía del lenguaje. Incluso, en uno de sus capítulos, Tom Grimwood ${ }^{10}$ reconstruye el argumento que Nietzsche propuso sobre la muerte de dios, con premisas y conclusiones, mostrando que, a pesar de las reticencias que tuvo Nietszche hacia la filosofía sistematizada en argumentos, su propuesta filosófica no está exenta.

Otro ejemplo lo encontramos en la obra de Charles Taylor, en la cual reconstruye una diversidad de argumentos que Hegel defiende en la Fenomenología del espíritu. Así, tanto en su libro Hegel ${ }^{11}$ como en su artículo de "Opening Arguments of the Phenomenology ${ }^{12}$ " él considera que Hegel expresa argumentos trascendentales para defender ciertas ideas que Immanuel Kant y Ludwig Wittgenstein defienden también respecto al conocimiento, a saber:

Todo conocimiento ha de poder ser conceptualizado.

Las razones que ofrece son las siguientes:

5 Welton, Donn (ed.). The Essential Husserl. Boomington: Indiana University Press. 1999.

6 Hegel, George Wilhelm Friedrich. La Fenomenología del espíritu. México: Fondo de Cultura Económica. 1966.

7 Gadamer, Hans-George. El giro hermenéutico. España: Cátedra. 1998.

8 Beuchot, Mauricio. Tratado de hermenéutica analógica. México: UNAM. 1997.

9 Bruce, Michael and Steven Barbone. Just the Arguments. 100 of the Most Important Arguments in Western Philosophy. United Kingdom: Blackwell. 2011.

10 Grimwood, Tom. "Nietzsche's Death of God". In Bruce, Michael and Steven Barbone. Just the Arguments. 100 of the Most Important Arguments in Western Philosophy. United Kingdom: Blackwell. 2011.

11 Taylor, Charles. Hegel. Cambridge: Cambridge University Press. 1975.

12 Taylor, Charles. "The Opening Arguments of the Phenomenology". In Alasdair MacIntyre (ed.). Hegel: A Collection of Critical Essays. Ney York: Doubleday. 1972. 
1. Los datos de los sentidos son insuficientes para que todo sujeto mantenga en su conciencia aquello que se le presenta.

2. No puede haber conocimiento fenoménico sin lenguaje ${ }^{13}$.

El valor de la propuesta exegética de Taylor yace en traducir a un lenguaje contemporáneo las diferentes propuestas epistémicas, éticas, estéticas, religiosas y políticas de Hegel. Esta propuesta es tan atractiva que muchos otros estudiosos lo han seguido en el camino, tales como Robert Stern ${ }^{14}$, Robert Pippin $^{15}$, Stephen Houlgate ${ }^{16}$, creando la Hegel Society of Great Britain ${ }^{17}$.

\section{La lógica, el análisis conceptual y la filosofía analítica}

La Hegel Society of Great Britain está formada, en su mayoría, por filósofas educadas y filósofos educados en la tradición analítica. Es propio de la filosofía analítica trabajar y evaluar argumentos explícitamente.

Como sabemos, la filosofía analítica comienza con cuatro filósofos emblemáticos: el alemán Gottlob Frege ${ }^{18}$, los británicos Bertrand Russel1 ${ }^{19}$ y George Edward Moore ${ }^{20}$, y el austriaco Ludwig Wittgenstein ${ }^{21}$. Uno de los objetos de estudio en los que estos estudiosos centraron su atención fue el lenguaje natural. Consideraron que los diversos problemas filosóficos se podían resolver o disolver a partir de un análisis lógico y lingüístico.

Los expertos comenzaron su análisis investigando las propiedades epistémicas de ciertas oraciones declarativas de identidad. Estas, en principio, eran oraciones semánticamente equivalentes, aunque había algo en sus propiedades que hacía pensar que podían tener algunos elementos semánticos diferentes, ya que unas eran informativas y ampliaban el conocimiento, pero otras no.

13 Taylor, Charles. "The Opening Arguments of the Phenomenology”, pp. 156-158.

14 Stern, Robert. Hegel and the Phenomenology of Spirit. London: Routledge. 2002.

15 Pippin, Robert. Hegel's Idealism. The Satisfaction of Self-consciousness. Cambridge: Cambridge University Press. 1989.

16 Houlgate, Stephen. The Opening of Hegel's Logic. From Being to Infinity. West Lafayette: Purdue University Press. 2006.

17 https://hegel-society.org.uk/.

18 Cfr. Frege, Gottlob. "Sense and Reference". The Philosophical Review. 57 (3). 1948 (1892).

19 Cfr. Russell, Bertand. "On Denoting”. Mind. 14. 1905.

20 Cfr. Moore, George Edward. Principia Ethica. Cambridge: Cambridge University Press. 1903.

21 Cfr. Wittgenstein, Ludwig. Tractatus Logico-Philosophicus. London: Routledge. 1922; Philosophical Investigations. Oxford: Blackwell. 1953. 
Los especialistas mencionados continuaron su análisis con temas epistemológicos, ontológicos, éticos, estéticos y metafísicos. Un ejemplo lo vemos en las Investigaciones Filosóficas de Wittgenstein. Interesado en qué consiste que alguien siga una regla, como la función de la suma, la aplicación de conceptos de colores o las señales de caminos en la carretera, consideró el concepto "entender" en contextos diversos ${ }^{22}$. La idea era encontrar un significado común, el cual compartieran todos los escenarios, para determinar cuándo cierta persona sigue una regla al entenderla. En este análisis, Wittgenstein evalúa, también, conceptos opuestos, como "mal entender" o no entender", con la intención de analizarlos y obtener información respecto al concepto que le interesa, dado que, según él, si queremos saber en qué consiste "entender", tenemos que hacer una exégesis de todos los conceptos que pueden relacionarse con este.

Con el análisis anterior, la contribución es diversa; una es la de no pensar los conceptos de manera aislada para comprenderlos; otra, evaluarlos en contextos, para entender cualquier tipo de tema; o, más importante aún, los ejemplos o experimentos mentales son relevantes para evaluar nuestras propuestas ${ }^{23}$.

Otro ejemplo interesante, aunque de naturaleza distinta, lo encontramos en el artículo de Russell "Sobre la denotación". En dicho ensayo, el autor realiza un análisis lógico de ciertas oraciones declarativas para mostrar que aquellas con nombres vacíos o descripciones definidas que sustituyen nombres, como "Hamlet habla con el espíritu de su padre", "la estrella de la mañana es brillante" o "el actual rey de Francia usa peluca", pueden ser entendidas usando tanto la negación como los cuantificadores universales y existenciales de la lógica clásica ${ }^{24}$. Así, para ejemplificar empleando un nombre vacío, podemos negar la anterior oración ficticia, diciendo que no hay una persona llamada Hamlet al que se le presenta el espíritu de su padre. Puesto que esa proposición es falsa, dado que no hay alguien llamado Hamlet, al ser un personaje ficticio, su negación es verdadera. Ese tipo de análisis ha contribuido a usar la lógica moderna para esclarecer, entre otras cosas, como lo mostraré en las secciones que siguen, qué contenido expresan ciertas

22 Wittgenstein, Ludwig. Philosophical Investigations, parágrafos 185-200.

23 Cfr. Comesaña, Juan Manuel. Lógica informal, falacias y argumentos filosóficos. Argentina: Eudeba. 2001

24 Russell, Bertand. "On Denoting”, pp. 479-481. 
oraciones cuyos sustantivos o descripciones definidas no están por un objeto o estado de cosas. También nos ha manifestado, mucho más importante aún, cuál es el papel de la verdad y la falsedad en dichas oraciones.

\section{¿En qué ha contribuido la tradición analítica a la filosofía?}

Usando la lógica o haciendo un análisis lingüístico, un considerable número de filósofas y filósofos del siglo XX y XXI han propuesto, analizado y evaluado argumentos sobre la mente humana ${ }^{25}$ y no-humana ${ }^{26}$; los usos diversos del lenguaje natural ${ }^{27}$; la estructura de las diferentes sociedades ${ }^{28}$; el género y la diversidad sexual ${ }^{29}$; la ética y la bioética ${ }^{30}$; la tecnología y las nuevas formas de comunicación ${ }^{31}$; los diferentes tipos de lógicas ${ }^{32}$, entre otros.

Sin embargo, no es exclusivo de la tradición analítica el uso de la lógica y el análisis lingüístico. Como vimos, Platón ya hacía análisis conceptual, al dialogar sistemáticamente con sus interlocutores, sobre el conocimiento, el amor, la verdad, el bien, la sociedad, etc. Todos sus argumentos, el análisis de ellos, los tenemos en la actualidad ${ }^{33}$. Es Platón, diría yo, el padre que nos ha enseñado a hacer filosofía usando y evaluando argumentos.

Otro filósofo importante es Aristóteles, el padre de la lógica. Él empleaba en sus escritos no solo un análisis conceptual, como el de la esencia o la existencia ${ }^{34}$, sino, igualmente, la lógica de las categorías para analizar y entender argumentos, la relación de inferencia que hay entre ellos, esquematizándolos, como:

1. Los hombres son mortales.

2. Sócrates es hombre.

25 Chalmers, David. The Conscious Mind. New York: Oxford University Press. 1996.

26 Nagel, Thomas. "What is it like to be a bat?". Philosophical Review. 83. 1974.

27 Austin, John. How to do things with words. Cambridge: Harvard University Press. 1962.

28 Rawls, John. A Theory of Justice. Cambridge: Harvard University Press. 1971.

29 Butler, Judith. Gender Trouble: Feminism and the Suversion of Identity. New York: Routledge. 1990.

30 Cfr. Engelhardt, Tristram. The Foundations of Bioethics. New York: Oxford University Press. 1996.

31 Mitcham, Carl. Thinking Through Technology: The Path Between Engineering and Philosophy. Chicago: University of Chicago Press. 1994.

32 Morado, Raymundo. “La formalización del sentido común”. En: María José Frápolli Sanz (coord.). Filosofía de la lógica. España: Tecnos. 2008.

33 Una detallada y amplia bibliografía se puede encontrar en el siguiente artículo: Kraut, Richard. "Plato". In The Stanford Encyclopedia of Philosophy. Edited by Edward N. Zelta. 2017.

34 Aristóteles. Metafisica. Madrid: Editorial Gredos. 1994. 
Por lo tanto, Sócrates es mortal.

Fue Aristóteles, así, quien nos legó el instrumento ${ }^{35}$ con el que trabajamos filósofas y filósofos para evaluar argumentos.

No obstante, la filosofía analítica ha sofisticado la lógica y el análisis conceptual, incrementando la primera en diferentes clases no-monotónicas ${ }^{36}$, o haciendo un examen de oraciones que no son solamente declarativas, sino también interrogativas o exclamativas ${ }^{37}$. Esto es su importancia y valor en nuestros días.

Más aun, la filosofía analítica nos ha enseñado que el uso de la lógica y del análisis lingüístico se aplica a argumentos, retomando, en mi opinión, la herencia platónica y aristotélica. Nos ha mostrado, asimismo, que, cualquiera que sea el método utilizado por la filósofa o el filósofo, pueden extraerse y evaluarse argumentos de las obras más emblemáticas en la historia de la filosofía.

Esta es la enseñanza, creería yo, de la filosofía analítica, a saber: el objeto de estudio de la filosofía son los argumentos, los cuales se evalúan o usando un dispositivo como la lógica o un dispositivo como el análisis conceptual.

\section{¿La lógica tiene alguna utilidad para la filosofía?}

La lógica moderna es un instrumento que fue pensado para hacer rigurosa a la filosofía. Ante la especulación, serviría para que los argumentos filosóficos tuvieran fundamento. El análisis lingüístico también seguía este objetivo, que la filosofía fuera rigurosa y pudiera fundamentar sus propuestas, como lo hacían la matemática, la física o la química. Independientemente de si la filosofía analítica ha sido exitosa con esta propuesta, creo que la lógica y el análisis conceptual son útiles, si consideramos que el objeto de estudio son los argumentos. En lo que resta del artículo, voy a mostrar esto.

Para poner un ejemplo, pensemos en la siguiente tesis ${ }^{38}$ :

(R) Una acción física o verbal $x$ es considerada violenta si y solo si $x$ rompe cierta regularidad o normatividad establecida por una comunidad.

35 Aristotle. The Organon, or Logical Treatises. Vol. I and II. London: George Bell and Sons. 1889 (copia electrónica).

36 MacCarthy, John. "Circumscription - A Form of Non-Monotonic Reasoning". Artificial Intelligence. 13. 1980.

37 Grice, Paul. "Logic and Conversation". In Donald Davison and G. Harmann (eds.). The Logic of Grammar. Encino, CA: Dickenson. 1975.

38 Esta tesis la defiendo en Suárez-Rivero, David. "Acciones, sistemas normativos o creencias. ¿Dónde se fundamenta la violencia?”. Principia: an International Journal of Epistemology. (25) 1. 2021. 
Una manera de formalizar lo anterior es de la manera explicada a continuación. Siendo mi ontología acciones físicas o verbales, y reglas o normas establecidas por cualquier comunidad indefinida, podemos comenzar especificando los predicados de modo formal:

Fx: $x$ es una acción física.

Bx: $x$ es una acción verbal.

Lx: $x$ es una acción violenta.

Rxz: x rompe cierta regularidad establecida por $\mathrm{z}$.

Nxz: $x$ rompe cierta normatividad establecida por $z$.

Podemos proporcionar, así, una vez definidos los predicados con sus variables, la siguiente formalización lógica, a la cual llamaré $\left(\mathrm{R}^{*}\right)$ :

$\left(\mathrm{R}^{*}\right)(\mathrm{x})(\mathrm{z})[((\mathrm{Fx} \vee \mathrm{Bx}) \rightarrow(\mathrm{Lx})) \leftrightarrow(\mathrm{Rxz} \vee \mathrm{Nxz})]$,

la cual dice:

Dada cualquier $x$ y cualquier $z$, si $x$ es una acción física o $x$ es una acción verbal, entonces $x$ es una acción violenta si y solo si $x$ rompe con cierta regularidad establecida por $z$ o $x$ rompe con cierta normatividad establecida por $z$.

¿Qué utilidad tiene traducir a un lenguaje formal la tesis $(\mathrm{R})$ como $\left(\mathrm{R}^{*}\right)$ ?

Primeramente, nos sirve para identificar los cuantificadores, los cuales determinan los objetos —el dominio del discurso — a los que se predica la idea defendida. Particularmente, nos permite identificar los cuantificadores universales mostrados por $\left(\mathrm{R}^{*}\right)$, los cuales no se muestran explícitamente en $(R)$, haciendo ver que es una tesis universal.

También nos permite identificar las aseveraciones simples y complejas, estas últimas constituidas por ciertos conectores del lenguaje natural, a saber: dos disyunciones, un condicional y un bicondicional, siendo este último el conector principal, cosa que no se aprecia a simple vista, una vez más, en (R). Hacer esto nos facilita entender cuáles son los compromisos de $\left(\mathrm{R}^{*}\right)$ con su verdad. En particular, podemos comprender que su compromiso es una equivalencia material, en la que tanto la fórmula de la derecha como la de la izquierda han de ser verdaderas para que la tesis también lo sea. 
Asimismo, retomar las dos ideas anteriores nos deja estructurar y asimilar la tesis que se defiende, al contemplar su universalidad y establecer que para que se dé una acción violenta han de cumplirse ciertos requisitos, a saber: que rompa una regla o norma. Si estos requisitos no se cumplen, entonces una acción no es violenta.

En otras palabras: dado que $(\mathrm{R})$ está formulada en $\left(\mathrm{R}^{*}\right)$ usando un bicondicional como conector principal, podemos comprender que si se da lo primero - i. e. una acción violenta-, entonces se da lo segundo - i. e. el rompimiento de una regla o norma-, pero si se da lo segundo - i. e. el rompimiento de una regla o norma-, entonces se da lo primero - i. e. una acción violenta - Esto quiere decir, también, que o una acción es violenta y rompe una regla o norma, o una acción no es violenta y no rompe una regla o norma.

Todas las paráfrasis anteriores nos las permite la lógica, al saber la equivalencia entre aseveraciones, la cual nos la proporciona sus reglas de remplazo $^{39}$. Normalmente, es importante contar con varias paráfrasis de la tesis que defendemos, ya que nos faculta entenderla ampliamente, viendo sus consecuencias. En el caso de $(\mathrm{R})$ formulada como $\left(\mathrm{R}^{*}\right)$, nuestras diversas paráfrasis nos indican que la relación entre una acción violenta y el rompimiento de reglas o normas es necesaria. Es decir, no puede darse la una sin la otra, idea bastante ambiciosa.

La formalización de una tesis nos permite, asimismo, contemplar objeciones. Por ejemplo, para objetar (R), si encontramos un caso en el cual hay rompimiento de regla o norma, pero no se considera violencia, entonces (R) es falsa. O, si hallamos otro caso en el que, además del rompimiento de regla o norma, se requiere un requisito extra para considerar una acción violenta, entonces mostramos que (R) es incompleta. Estas dos objeciones, cuyas respuestas pueden encontrarse en mi artículo mencionado en la nota al pie de página, de no tener respuesta, mostrarían que la relación de necesidad entre una acción violenta y el rompimiento de regla o norma no se cumple. Así, si eso fuera cierto, habría que o rechazar (R) o replantearse. Estos son los

39 Copi, Irving M. Symbolic Logic. London: Collier MacMillan Publishers. 1973; Copi, Irving M., Carl Cohen and Kenneth MacMahon. Introduction to Logic. Edinburgh: Pearson. 2014. 
beneficios que nos da la lógica cuando hacemos filosofía ${ }^{40}$ y ello se muestra cuando traducimos a $(\mathrm{R})$ como $\left(\mathrm{R}^{*}\right)$.

\section{¿Hay más beneficios al usar la lógica?}

La lógica nos proporciona más beneficios, los cuales podemos ver al realizar la demostración de $\left(\mathrm{R}^{*}\right)$. Inicialmente, podemos recurrir a una derivación, asumiendo que $\left(\mathrm{R}^{*}\right)$ es una tautología. Si lo es, entonces observamos que $\left(\mathrm{R}^{*}\right)$ no depende de premisa alguna $\mathrm{o}$, si se quiere, $\left(\mathrm{R}^{*}\right)$ es un argumento cuya conclusión es ella misma. Veamos si esto es el caso.

Como el conector principal es un bicondicional, podemos intentar una prueba indirecta. Para ello, tenemos que suponer como premisa que $\left(\mathrm{R}^{*}\right)$ no es verdad, encontrar una contradicción en la derivación y, con esto, mostrar que $\left(\mathrm{R}^{*}\right)$ es válida — i. e. que no es verdad que su negación es el caso- - La derivación lógica es la siguiente, en la cual la línea 1 es la premisa asumida del argumento y lo que sigue al símbolo // su conclusión:

1. (x) $(\mathrm{z}) \sim[((\mathrm{Fx} \vee \mathrm{Bx}) \rightarrow(\mathrm{Lx})) \leftrightarrow(\mathrm{Rxz} \vee \mathrm{Nxz})]$

$/ /(\mathrm{x})(\mathrm{z})[((\mathrm{Fx} \vee \mathrm{Bx}) \rightarrow(\mathrm{Lx})) \leftrightarrow(\mathrm{Rxz} \vee \mathrm{Nxz})]$

2. $(\mathrm{z}) \sim[((\mathrm{Fa} \vee \mathrm{Ba}) \rightarrow(\mathrm{La})) \leftrightarrow(\mathrm{Raz} \vee \mathrm{Naz})]$

3. $\sim[((\mathrm{Fa} \vee \mathrm{Ba}) \rightarrow(\mathrm{La})) \leftrightarrow(\mathrm{Rac} \vee \mathrm{Nac})]$
2, U.I.
4. $\sim[((\mathrm{Fa} \vee \mathrm{Ba}) \rightarrow(\mathrm{La})) \cdot(\mathrm{Rac} \vee \mathrm{Nac})] \vee \sim[\sim((\mathrm{Fa} \vee \mathrm{Ba}) \rightarrow(\mathrm{La})) \cdot \sim(\mathrm{Rac}$ $\vee \mathrm{Nac})]$

3, Equiv.

5. $\sim[[((\mathrm{Fa} \vee \mathrm{Ba}) \rightarrow(\mathrm{La})) \cdot(\mathrm{Rac} \vee \mathrm{Nac})] \cdot[\sim((\mathrm{Fa} \vee \mathrm{Ba}) \rightarrow(\mathrm{La})) \cdot \sim(\mathrm{Rac}$ $\vee \mathrm{Nac})]]$

4, De M.

6. $\sim[[((\mathrm{Fa} \vee \mathrm{Ba}) \rightarrow(\mathrm{La})) \cdot(\mathrm{Rac} \vee \mathrm{Nac})] \cdot[\sim(\mathrm{Rac} \vee \mathrm{Nac}) \cdot \sim((\mathrm{Fa} \vee \mathrm{Ba})$

$\rightarrow(\mathrm{La}))]]$

5, Com.

40 Otros beneficios pueden encontrarse en Hernández Ortiz, Héctor. “¿Ayuda la enseñanza de la lógica a los estudiantes a argumentar mejor?”. Quadripartita Ratio. 2(3). 2017. Agradezco a Héctor la discusión sostenida desde el 2019, cuando Víctor Cantero-Flores me invitó a la Universidad del Caribe, en México, a hablar sobre estos temas. También agradezco a un corrector de estilo de la Revista Praxis las sugerentes recomendaciones a una versión previa de este ensayo. 
7. $\sim[[((\mathrm{Fa} \vee \mathrm{Ba}) \rightarrow(\mathrm{La})) \cdot((\mathrm{Rac} \vee \mathrm{Nxc}) \cdot \sim(\mathrm{Rac} \vee \mathrm{Nxc}))] \cdot[\sim((\mathrm{Fa} \vee \mathrm{Ba})$ $\rightarrow(\mathrm{La}))]]$

6, Assoc.

8. $\sim[[((\mathrm{Rac} \vee \mathrm{Nxc}) \cdot \sim(\mathrm{Rac} \vee \mathrm{Nxc})) \cdot((\mathrm{Fa} \vee \mathrm{Ba}) \rightarrow(\mathrm{La}))] \cdot[\sim((\mathrm{Fa} \vee \mathrm{Ba})$ $\rightarrow(\mathrm{La}))]]$

7, Com.

9. $\sim[((\mathrm{Rac} \vee \mathrm{Nxc}) \cdot \sim(\mathrm{Rac} \vee \mathrm{Nxc})) \cdot[((\mathrm{Fa} \vee \mathrm{Ba}) \rightarrow(\mathrm{La})) \cdot[\sim((\mathrm{Fa} \vee \mathrm{Ba})$ $\rightarrow(\mathrm{La}))]]]$

8, Assoc.

10. $\sim((\mathrm{Rac} \vee \mathrm{Nxc}) \cdot \sim(\mathrm{Rac} \vee \mathrm{Nxc})) \vee \sim[((\mathrm{Fa} \vee \mathrm{Ba}) \rightarrow(\mathrm{La})) \cdot[\sim((\mathrm{Fa} \vee \mathrm{Ba})$ $\rightarrow(\mathrm{La}))]]$

9, De M.

11. $\sim[((\mathrm{Fa} \vee \mathrm{Ba}) \rightarrow(\mathrm{La})) \cdot[\sim((\mathrm{Fa} \vee \mathrm{Ba}) \rightarrow(\mathrm{La}))]] \vee \sim((\mathrm{Rac} \vee \mathrm{Nxc}) \cdot \sim$ $(\operatorname{Rac} \vee N x c))$

10, Com.

12. $((\mathrm{Fa} \vee \mathrm{Ba}) \rightarrow(\mathrm{La})) \cdot[\sim((\mathrm{Fa} \vee \mathrm{Ba}) \rightarrow(\mathrm{La}))] \rightarrow \sim((\mathrm{Rac} \vee \mathrm{Nxc}) \cdot \sim(\mathrm{Rac}$ $\vee \mathrm{Nxc})$ )

11, Impl.

13. $((\mathrm{Fa} \vee \mathrm{Ba}) \rightarrow(\mathrm{La})) \cdot[\sim((\mathrm{Fa} \vee \mathrm{Ba}) \rightarrow(\mathrm{La}))]$ C.P.

14. $\sim((\operatorname{Rac} \vee N x c) \cdot \sim(\operatorname{Rac} \vee N x c)) 12$, 13, M.P.

Como puede apreciarse ya en la línea 5 y, sobre todo, en la 14, asumir la negación de $\left(\mathrm{R}^{*}\right)$ no nos lleva a ninguna contradicción. Por el contrario, lo que dichas líneas nos dicen es que la afirmación de una oración compuesta y su negación no es el caso, al asumir como falsa $\left(\mathrm{R}^{*}\right)$. Por lo tanto, $\left(\mathrm{R}^{*}\right)$ no es una tautología, lo cual significa que no depende de sí misma, sino que han de haber ciertas premisas explícitas que la apoyan. Así, una forma de leer a $\left(\mathrm{R}^{*}\right)$ sería siendo la conclusión de las siguientes dos premisas, cuyo conector principal es un condicional.

Primera premisa: Dada cualquier $x$ y cualquier $z$, si $x$ es una acción física o $x$ es una acción verbal, entonces $x$ es una acción violenta solo si $x$ rompe con cierta regularidad establecida por $z$ o $x$ rompe con cierta normatividad establecida por $z$. La manera de formalizarla sería:

$(\mathrm{x})(\mathrm{z})[((\mathrm{Fx} \vee \mathrm{Bx}) \rightarrow(\mathrm{Lx})) \rightarrow(\mathrm{Rxz} \vee \mathrm{Nxz})]$ 
Segunda premisa: Dada cualquier $x$ y cualquier $z$, si $x$ rompe con cierta regularidad establecida por $z$ o $x$ rompe con cierta normatividad establecida por $z$, entonces si $x$ es una acción física o $x$ es una acción verbal se sigue que $x$ es una acción violenta. El modo de formalizarla sería:

$(\mathrm{x})(\mathrm{z})[(\mathrm{Rxz} \vee \mathrm{Nxz}) \rightarrow((\mathrm{Fx} \vee \mathrm{Bx}) \rightarrow(\mathrm{Lx}))]$

Conclusión: Dada cualquier $x$ y cualquier $z$, si $x$ es una acción física o $x$ es una acción verbal, entonces $x$ es una acción violenta si y solo si $x$ rompe con cierta regularidad establecida por $z$ o $x$ rompe con cierta normatividad establecida por $z$. Su formalización es la que ya conocemos, a la cual hemos llamado $\left(\mathrm{R}^{*}\right)$ :

$(\mathrm{x})(\mathrm{z})[((\mathrm{Fx} \vee \mathrm{Bx}) \rightarrow(\mathrm{Lx})) \leftrightarrow(\mathrm{Rxz} \vee \mathrm{Nxz})]$

Su demostración es la que viene, en la que las líneas 1 y 2 son las premisas y la conclusión es la fórmula lógica que sigue al signo //:

1. $(\mathrm{x})(\mathrm{z})[((\mathrm{Fx} \vee \mathrm{Bx}) \rightarrow(\mathrm{Lx})) \rightarrow(\mathrm{Rxz} \vee \mathrm{Nxz})]$

2. (x) (z) $[(\mathrm{Rxz} \vee \mathrm{Nxz}) \rightarrow((\mathrm{Fx} \vee \mathrm{Bx}) \rightarrow(\mathrm{Lx}))]$

$/ /(\mathrm{x})(\mathrm{z})[((\mathrm{Fx} \vee \mathrm{Bx}) \rightarrow(\mathrm{Lx})) \leftrightarrow(\mathrm{Rxz} \vee \mathrm{Nxz})]$

3. $(\mathrm{z})((\mathrm{Fa} \vee \mathrm{Ba}) \rightarrow(\mathrm{La})) \rightarrow(\mathrm{Raz} \vee \mathrm{Naz})$ 1, U.I.

4. $((\mathrm{Fa} \vee \mathrm{Ba}) \rightarrow(\mathrm{La})) \rightarrow(\mathrm{Rac} \vee \mathrm{Nac}) 3$, U.I.

5. (z) $(\mathrm{Raz} \vee \mathrm{Naz}) \rightarrow((\mathrm{Fa} \vee \mathrm{Ba}) \rightarrow(\mathrm{La})) 2$, U.I.

6. $(\mathrm{Rac} \vee \mathrm{Nac}) \rightarrow((\mathrm{Fa} \vee \mathrm{Ba}) \rightarrow(\mathrm{La})) 5$, U.I.

7. $((\mathrm{Fa} \vee \mathrm{Ba}) \rightarrow(\mathrm{La})) \rightarrow(\mathrm{Rac} \vee \mathrm{Nac}) \cdot(\mathrm{Rac} \vee \mathrm{Nac}) \rightarrow((\mathrm{Fa} \vee \mathrm{Ba}) \rightarrow(\mathrm{La}))$

4, 6, Conj.

8. $((\mathrm{Fa} \vee \mathrm{Ba}) \rightarrow(\mathrm{La})) \leftrightarrow(\mathrm{Razc} \vee \mathrm{Nac}) 7$, Equiv.

9. $(\mathrm{x})[((\mathrm{Fx} \vee \mathrm{Bx}) \rightarrow(\mathrm{Lx})) \leftrightarrow(\mathrm{Rxc} \vee \mathrm{Nxc})] 8$, U.G.

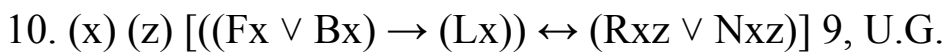

Como se aprecia en esta demostración, al ser la conclusión de un argumento, $\left(\mathrm{R}^{*}\right)$ resulta consecuencia lógica de las dos premisas anteriores, lo cual indica que el argumento es válido. Es decir, de ser verdaderas las premisas, lo es también la conclusión. 
En muchas ocasiones, las premisas de los argumentos están implícitas, por lo que se vislumbra solamente la conclusión. Lo que uno estila hacer es lo que realizamos anteriormente, a saber: se trata de determinar si dicha conclusión es una tautología, en la que ella sea la única premisa del argumento. Si no lo es, hay que buscar probables premisas implícitas, muchas veces interpretando lo que dice la autora o el autor, o aportándolas nosotras mismas o nosotros mismos. En el caso de $\left(\mathrm{R}^{*}\right)$, las premisas formuladas pudieron respaldarla, haciendo que la conclusión se siguiera de ellas, mostrando con ello su validez.

\section{Conclusión}

¿La lógica hace de la filosofía una disciplina científica? Si pensamos que toda ciencia tiene instrumentos de los que se sirve para ampliar el sistema de conocimiento de manera confiable, la lógica hace que la filosofía sea una disciplina científica, al brindarle condiciones para que la nueva información que obtiene lo sea.

Hay, sin embargo, dos objeciones a la anterior idea. La primera es que los resultados a los que llega la filosofía difícilmente son concluyentes, como podrían ser los de la matemática o la física. La segunda consiste en que no es de mutuo acuerdo que la lógica sea su instrumento, como sí lo es la probabilidad para la economía.

Es verdad que los resultados de la filosofía no son concluyentes. No obstante, esto no quiere decir, que no sea científica. Dado que lo que yace al fondo de proporcionar y evaluar argumentos es buscar las condiciones de posibilidad de fundamentar cualquier asunto, parte de su naturaleza es identificar cualquier problema en lo que se asevera. Esto hace a la rama filosófica, frente a las demás disciplinas, propedéutica, en el sentido de que se puede reflexionar sobre cualquier tema, inclusive sobre las tesis de otras áreas científicas, con la finalidad de indagar su fundamento. Así, lo que hace la filosofía, al proporcionar y evaluar argumentos, es permanecer en la búsqueda de las mejores condiciones de fundamentación, lo cual es arduo y polémico.

Ahora bien, ¿el que los resultados de la filosofía no sean concluyentes quiere decir que esta no proporciona nueva información al sistema de conocimiento? En absoluto. En su infatigable labor de ofrecer y evaluar 
argumentos, va obteniendo resultados mínimos, los cuales otorgan datos novedosos. Por ejemplo, últimamente, los conceptos de "mujer" y "hombre" han cambiado, tras una gran discusión sobre qué significa ser mujer y hombre. La tendencia es robustecer el significado de dichos conceptos e incluir a mujeres y hombres transgénero. Este resultado no se habría dado sin la larga discusión de Judith Butler ${ }^{41}$.

Otro ejemplo es el de volver inclusivo el uso del lenguaje. Antes, solamente se usaba el género masculino para dirigirse a mujeres y hombres. Hoy se está optando o por usar tanto el masculino como el femenino o incorporar la letra "e" en todas aquellas palabras que puedan incluir a "ambes". Este resultado no se habría logrado sin la contribución a lo discutido por Janice Moulton $^{42}$ o Adele Mercier ${ }^{43}$. Esto muestra, así, que la filosofía provee nuevo conocimiento, el cual puede, incluso, modificar el comportamiento.

¿Qué sucede con la segunda objeción? Es cierto, no hay común acuerdo. ¿Esto quiere decir que la lógica no es un instrumento de la filosofía? En absoluto. Como he mostrado, muchas filósofas y muchos filósofos la usan en sus argumentos. Inclusive, puede traducirse a un análisis lógico lo que hacen otras filósofas y otros filósofos que no la incorporan, cosa que no ha hecho esta comunidad filosófica con la manera que tiene la lógica de expresar ciertos contenidos. Ello pone, de tal modo, en ventaja a la lógica y puede considerársele una herramienta general, ya que se muestra que con esta se puede trabajar cualquier contenido filosófico.

Considérese, asimismo, que la lógica permite claridad, mayor entendimiento y un alto grado de confiabilidad en los argumentos que se defienden. Si a esto puede llamársele científico, entonces, una parte de la filosofía lo es, la cual no excluye a aquella que no la usa, dado que, como revelé, todo contenido puede formularse en términos lógicos, haciendo de la lógica un instrumento de la filosofía.

41 Butler, Judith. Gender Trouble: Feminism and the Suversion of Identity. New York: Routledge. 1990.

42 Moulton, Janice. "The Myth of the Neutral "Man"”. In Mary Vetterling-Bragin (1981), Sexist Language: A Modern Philosophical Analysis. Totowa, NJ: Littlefield and Adams. 1981.

43 Mercier, Adele. "A Perverse Case of the Contingent A Priori: On the Logic of Emasculating Language". Philosophical Topics, 23 (2). 1995. 


\section{Bibliografía}

Aristóteles. Metafísica. Madrid: Editorial Gredos, 1994.

Aristoteles. The Organon or Logical Treatises of Aristotle: With Copious Elucidations from the Commentaries of Ammonius, Porphyry and Simplicius. Vol. 1 y 2. 20. London: George Bell and Sons, 1889.

Austin, J. L. How to Do Things with Words. Cambridge, Mass: Harvard Univ. Press, 1962.

Beuchot, Mauricio. Tratado de hermenéutica analógica. México: Facultad de Filosofía, Universidad Nacional Autónoma de México, 1997.

Bruce, Michael, and Steven Barbone, eds. Just the Arguments: 100 of the Most Important Arguments in Western Philosophy. Chichester, West Sussex, U.K: Wiley-Blackwell, 2011.

Butler, Judith. Gender Trouble: Feminism and the Subversion of Identity. Thinking gender. New York: Routledge, 1990.

Carroll, Noël, and Jinhee Choi, eds. Philosophy of Film and Motion Pictures: An Anthology. Blackwell philosophy anthologies. Malden, MA: Blackwell Pub, 2006.

Chalmers, David John. The Conscious Mind: In Search of a Fundamental Theory. Philosophy of mind series. New York: Oxford University Press, 1996.

Comesaña, Juan Manuel. Lógica informal, falacias y argumentos filosóficos. Buenos Aires: Eudeba, 2001.

Copi, Irving M. Symbolic Logic. London: Collier MacMillan Publishers, 1973.

Copi, Irving M., Carl Cohen, and Kenneth MacMahon. Introduction to Logic. 14 ed., Pearson new international ed. Pearson custom library. Harlow: Pearson Education Limited, 2014.

Engelhardt, H. Tristram. The Foundations of Bioethics. 2nd ed. New York: Oxford University Press, 1996.

Frápolli Sanz, María José. Filosofía de la lógica. Madrid: Tecnos, 2008.

Frege, Gottlob. “Sense and Reference.” The Philosophical Review 57, no. 3 (May 1948): 209.

Gadamer, Hans-Georg. El Giro Hermenéutico. Madrid: Ediciones Cátedra, 1998.

Grice, Paul. "Logic and Conversation." In Donald Davison and G. Harmann The Logic of Grammar. The Dickenson series in philosophy. Encino, Calif: Dickenson Pub. Co, 1975.

Grimwood, Tom. "Nietzsche's Death of God." In Just the Arguments, edited by Michael Bruce and Steven Barbone, 52-56. Oxford, UK: Wiley-Blackwell, 2011.

Hegel, Georg Wilhelm Friedrich, and Gustavo Leyva. Fenomenología del espíritu. Distrito Federal: FCE - Fondo de Cultura Económica, 1996.

Houlgate, Stephen. The Opening of Hegel's Logic: From Being to Infinity. Purdue University Press series in the history of philosophy. West Lafayette, Ind: Purdue University Press, 2006.

Husserl, Edmund, and Donn Welton. The Essential Husserl: Basic Writings in Transcendental Phenomenology. Studies in Continental thought. Bloomington, IN: Indiana University Press, 1999. 
MacIntyre, Alasdair C., ed. Hegel: A Collection of Critical Essays. Ney York: Doubleday, 1972.

McCarthy, John. "Circumscription-A Form of Non-Monotonic Reasoning." Artificial Intelligence 13, no. 1-2 (April 1980): 27-39.

Mercier, Adèle and University of Arkansas Press. "A Perverse Case of the Contingent A Priori: On the Logic of Emasculating Language (A Reply to Dawkins and Dummett)." Philosophical Topics 23, no. 2 (1995): 221-259.

Mitcham, Carl. Thinking through Technology: The Path between Engineering and Philosophy. Chicago: University of Chicago Press, 1994.

Moore, G. E., and Thomas Baldwin. Principia Ethica. Cambridge University Press, 1903.

Nagel, Thomas. "What Is It Like to Be a Bat?" The Philosophical Review 83, no. 4 (October 1974): 435.

Nietzsche, Friedrich. Así Hablo Zaratustra: Un Libro Para Todos y Para Nadie. Madrid: Alianza Editorial, 1953.

Pippin, Robert B. Hegel's Idealism: The Satisfactions of Self-Consciousness. Cambridge; New York: Cambridge University Press, 1989.

Plato, John M. Cooper, and D. S. Hutchinson. Complete Works. Indianapolis, Ind: Hackett Pub, 1997.

Rawls, John. A Theory of Justice. Cambridge: Harvard University Press. , 1971.

Russell, Bertrand. “On Denoting.” Mind 14 (1905): 479-493.

Stern, Robert and MyiLibrary. Routledge Philosophy Guidebook to Hegel and the Phenomenology of Spirit. London; New York: Routledge, 2002.

Taylor, Charles. Hegel. Cambridge: Cambridge Univ. Press, 1975.

Vetterling-Braggin, Mary, ed. Sexist Language: A Modern Philosophical Analysis. Littlefield Adams quality paperback series; 353. Totowa, N.J: Littlefield, Adams, 1981.

Wittgenstein, Ludwig. Tractatus Logico-Philosophicus. Oxford: Blackwell, 1953.

—. Tractatus Logico-Philosophicus. Routledge great minds. London; New York: Routledge, 2014.

Wittgenstein, Ludwig. Philosophical Investigations. New York: Macmillan, 1953. 